\title{
Mental Health Stigma: Attitudes of Physiotherapy Students and the Role of Education in Attitude Development
}

\section{Bev Sarin MSc MCSP1, Nathalie Brodie BSc (Hons) MCSP2 \\ ${ }^{1}$ Department of Physiotherapy, School of Healthcare Studies, Cardiff University \\ 2Department of Physiotherapy, Cardiff and Vale NHS Trust}

\section{Introduction}

Statistics show that 1 in 4 people will be affected by a Mental Health problem at some point in their lives (Rose, 2003). Physiotherapists play a diverse role throughout the Healthcare system, where people with Mental Health problems and disorders will be encountered in many settings, not solely in the Mental Health Services.

Physiotherapy students are the next generation of qualified therapists. As such their undergraduate education and experience needs to be focused on challenging any potential stigma towards engaging with people with Mental Health Disorders. This may be due to lack of understanding or anxiety, leading to prejudicial views gained prior to entering university.

Education needs to support the knowledge and skill development that is necessary to empower students, to allow them are to become capable of effective engagement and management of people with Mental Health Disorders.

An understanding of students' attitudes towards people with Mental Health Disorders can be used to shape design and delivery of the curriculum of undergraduate courses to make sure this is addressed.

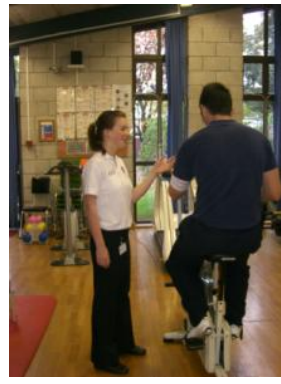

Clinical placements allow students to gain first hand experience of working with people with Mental Health Disorders

\section{Aim}

To Identify the attitudes that level 1 and level 3 physiotherapy students have towards people with Mental Health Disorders

To identify the role that clinical experience and the level 2 Mental Health teaching block play in influencing student attitudes towards people with Mental Health Disorders.

Challenging Stigma through Undergraduate Education

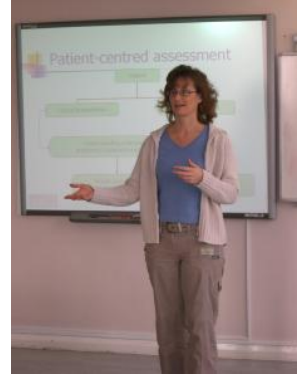

\section{Method}

40 level 1 and 40 level 3 students from the Physiotherapy BSc (Hons) course at Cardiff University

(from cohort sizes of 85 and 91 respectively)

volunteered to participate in the study.

Level 1 students were pre-clinical, whereas level 3 students had undertaken the Mental Health teaching block and 6 months clinical experience, which for some included a Mental health placement.

A questionnaire was used, containing closed ordinal point questions for attitude frequency, and open questions where further explanation was required.

Themes explored included

๑ Past experience of Mental Health Disorders

- Sources of influence on attitude development

- Experience of Mental Health clinical placements.

Ethical approval was gained through the School of Healthcare Studies Research Ethics Committee.

Analysis of frequency data associations was made using SPSS cross-tabulation and Chi-squared calculations. Thematic analysis was used on open questions.

\section{Results}

74 questionnaires were completed and returned, $n=37$ level 1 and $n=37$ level 3 .

$74 \%$ of level 1 students and $70 \%$ of level 3 students demonstrated attitudes within the positive range of the Likert scales, towards people with Mental Health Disorders.

On further questioning, students identified that the Media $(p=0.005)$ and Education $(p=0.002)$ played significant roles in influencing their attitudes.

Only 4 (level 3) students had undertaken a Mental Health placement; all of whom indicated a more positive view of people with Mental Health Disorder after their placements.

$25 \%$ of level 3 students reported a positive change in attitude following the Mental Health teaching block. No students reported a negative change of attitude following the teaching block.

Clinical Educators play a vital role in supporting the student's

experience and development in this area.

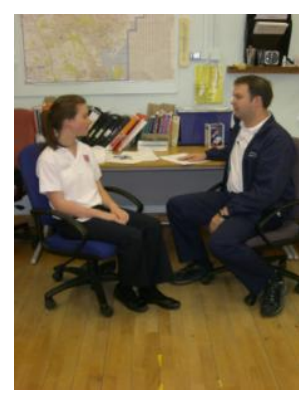

\section{Conclusion}

Education and the media were found to provide the most influence on attitudes of undergraduate physiotherapy students.

Students' attitudes were generally positive, and these were further enhanced by the Mental Health teaching block and clinical placements as part of their undergraduate training.

Although the results of students' attitudes to people with Mental Health Disorder were found to be in the positive side of the measurement scale used, these tended to fall in the 'slightly positive' rather than 'very positive' part of the range. Although this would indicate that students have a more positive than negative attitude towards people with Mental Health Disorder, further exploration of how positive that attitude is, may be indicated.

Both the Mental Health teaching block and clinical experience acted to influence the shift of attitudes in a positive direction.

\section{Implications}

The inclusion of Mental Health specific teaching within undergraduate physiotherapy training provides an essential support for students in the development of a more positive attitude, with resultant reduction in stigma, towards the engagement with clients with Mental Health Disorders.

In assisting positive changes in attitudes through the development of knowledge, understanding, skills and experience, providing increased confidence and awareness of patient needs, the physiotherapist of the future is better placed to provide 'Best Care' for patients, and act as role models to others within their working environment.

How does this improvement in attitude towards people with Mental Health Disorders at undergraduate level transfer into practice at postgraduate level? If it does, is there a resultant impact on the ability of this patient population to gain effective healthcare through generic services? Further investigation is needed.

\section{References}

Rose N (2003) Mental Health Disorder: A biomedical approach in: Everett T, Donaghy M, Feaver S. Interventions for Mental Health: An Evidence-based Approach for Physiotherapists and Occupational Therapists. Edinburgh, Butterworth Heinemann.

\section{Contact}

Department of Physiotherapy, School of Healthcare Studies, Cardiff University, Ty Dewi Sant, Heath Park Cardiff, Wales, UK. CF14 4XN

sarinbj@cardiff.ac.uk 